\title{
Kinematics of the Galactic Bulge from Radial Velocity and Proper Motion Optical Surveys
}

\author{
Céline Reylé $\hat{1}^{1,2}$, Annie C. Robin ${ }^{1}$, and Sébastien Picaud ${ }^{1}$ \\ ${ }^{1}$ Observatoire de Besançon, BP 1615, 25010 Besançon Cedex, France \\ ${ }^{2}$ E-mail: celine@obs-besancon.fr
}

Received 2003 September 30, accepted 2004 February 9

\begin{abstract}
Large-scale surveys provide new constraints on the structure and evolution of the Milky Way. The population synthesis approach is a useful tool to interpret such data sets and to test scenarios of evolution of the Galaxy. New constraints on Galactic parameters have been obtained from the Besançon model of population synthesis, in particular in the inner regions, thanks to near-infrared surveys less affected by interstellar extinction than the optical ones. We present here a few preliminary comparisons between observed and simulated distributions of proper motions in the direction of the Galactic bulge. Next we discuss how bulge stars can be observed and separated from other populations with the RAVE and Gaia surveys.
\end{abstract}

Keywords: Galaxy: bulge — Galaxy: kinematics and dynamics — surveys

\section{Introduction}

Recent or ongoing large-scale surveys at different wavelengths, such as SDSS and CFHTLS in the visible, and DENIS and 2MASS in the near-infrared, bring new constraints on the structure and the history of the Milky Way. In the visible range, the interstellar matter distributed in the plane is a major obstacle to the observation of the stars, particularly in the direction of the bulge and the Galactic centre.

The new surveys at near-infrared wavelengths, less sensitive to the interstellar extinction, allow us to probe the innermost regions of the Galaxy. The DENIS survey has produced specific observations in the direction of the bulge (G. Simon et al., in preparation). Picaud et al. (2003) used these data and the population synthesis model of Besançon (Robin et al. 2003) to adjust the density law parameters of the bulge.

Still, the kinematic modelling of the bulge has to be improved, as only few measurements of proper motions and radial velocities were available up to now for bulge stars. Recently, Peyaud et al. (2003) have obtained radial velocities for about 500 new planetary nebulae in the bulge, discovered from the MASH survey (Parker \& Phillipps 2001). Their spatial distribution and kinematics should bring constraints on the bulge dynamics. Moreover, Sumi et al. (2004) have released a proper motion catalogue for 5 million stars in the direction of the bulge, obtained over $11 \mathrm{deg}^{2}$ from OGLE-II data. This catalogue, as well as ongoing and forthcoming surveys such as RAVE and Gaia, will help in increasing our knowledge of the bulge kinematics.

In Section 2, we present the bulge kinematics as considered in the Besançon model of the Galaxy and compare a simulation from the model with the OGLE-II catalogue of stellar proper motions. In Sections 3 and 4, we use simulations to compute stellar densities and to predict if, and where, bulge stars will be observed with RAVE and Gaia. Discussions and details of further work are given in Section 5.

\section{Kinematics of the Bulge from OGLE-II Proper Motions}

The Besançon model of the Galaxy is based on a semiempirical approach, where physical constraints and our current knowledge of the formation and evolution of the Milky Way are used as a first approximation for the population synthesis. Then the free parameters are tuned to match solar neighbourhood data as well as deep star counts in many different colours and directions. The latest version of the model is described in Robin et al. (2003), where comparisons with various data are shown. (One can also perform specific simulations via the Web. ${ }^{1}$ )

Sumi et al. (2004) have measured the proper motions for stars in 49 fields in the direction of the Galactic bulge, covered many times by the OGLE-II experiment over a period of almost four years. The left panel in Figure 1 shows the colour-magnitude diagram for the SC5 field, towards the direction $l=-0.23^{\circ}, b=-1.33^{\circ}$. The right panel shows the predicted diagram from the Besançon model, taking into account the observational errors. The stars with $V-I \geq 5.5$ in the simulation are fainter than the observed ones. This might be due to inaccuracies in the evolutionary tracks used in the model for late M-giants and AGB stars, as the late stages of stellar

\footnotetext{
${ }^{1}$ http://www.obs-besancon.fr/modele/model2003.html
} 
evolution are not very well understood. This should be revised in the near future but has no effect on the present study. According to the simulation, the bulge stars have $V-I \geq 3$. Selecting stars redder than $V-I=3$ allows us to reject the foreground stars, which are mainly disk dwarf stars. The remainder are dominated by bulge giants, with a smaller contribution from disk giants.

The $\mu_{l}$ and $\mu_{b}$ distributions for all stars redder than $V-I=3$ are plotted in Figure 2. The black lines show the data distributions and the grey lines the simulated distributions. The bulge velocity dispersions used in the model are $\sigma_{U}=113 \mathrm{~km} \mathrm{~s}^{-1}, \sigma_{V}=115 \mathrm{~km} \mathrm{~s}^{-1}$, and $\sigma_{W}=100 \mathrm{~km} \mathrm{~s}^{-1}$. The bulge asymmetric drift is $v_{\text {ad }}=79 \mathrm{~km} \mathrm{~s}^{-1}$. These parameters allow us to reproduce the observed distributions reasonably well, although the errors might be underestimated. However, the precision of the proper motions is 0.1 arcsec century $^{-1}$, which corresponds to a precision of roughly $40 \mathrm{~km} \mathrm{~s}^{-1}$ on the velocity at $8 \mathrm{kpc}$, which is too large to derive accurate kinematics parameters.

Alard (2003) used a subtraction image method to measure proper motions in a small sub-area near the centre of one of the OGLE-II fields. He obtained a precision three times better. One then should be able to constrain
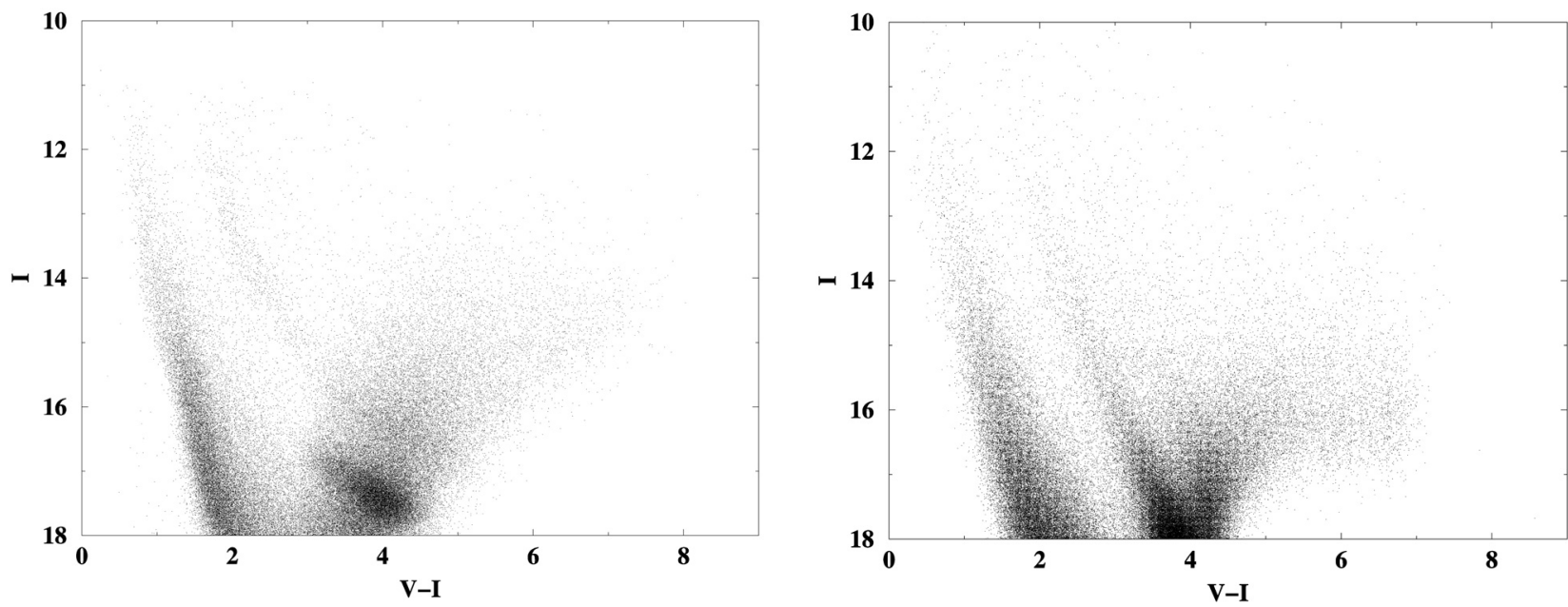

Figure 1 Colour-magnitude diagram for the OGLE-II SC5 field in the direction $l=-0.23^{\circ}, b=-1.33^{\circ}$. Left: data. Right: simulation.
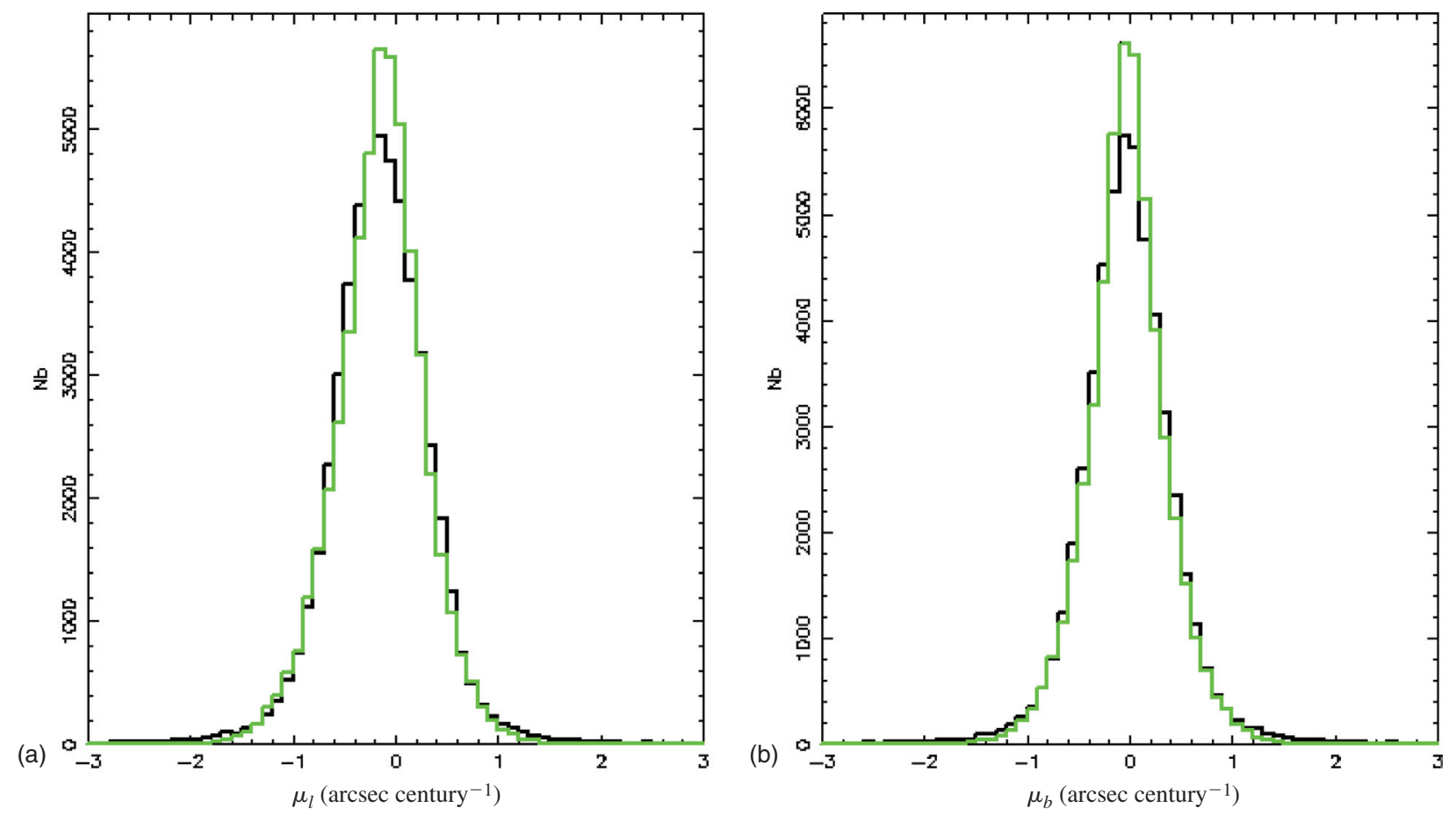

Figure $2 \mu_{l}$ (left) and $\mu_{b}$ (right) distributions for the stars with $V-I \geq 3$ in the OGLE-II SC5 field in the direction $l=-0.23^{\circ}, b=-1.33^{\circ}$. Black: data. Grey: simulation. 
the bulge kinematics parameters. Alard (2003) derived the following dispersions of the bulge stars: $\sigma_{V}=105 \mathrm{~km} \mathrm{~s}^{-1}$, $\sigma_{W}=82 \mathrm{~km} \mathrm{~s}^{-1}$.

\section{The Bulge, as Seen by RAVE}

The RAdial Velocity Experiment, RAVE, is a spectroscopic survey conducted with the UK Schmidt telescope at the AAO. It aims at providing radial velocities and metallicities for all stars up to $V=16$ (Steinmetz 2003). We used the model of Besançon to predict the number of bulge stars that will be detected by RAVE. The only limitation in observing bulge stars is the interstellar extinction. Table 1 gives the expected density of bulge stars at longitude $l=1^{\circ}$ and latitudes $|b|$ from $0^{\circ}$ to $5^{\circ}$, assuming different values for interstellar extinction.

Table 1. Density of bulge stars up to $V=16$ predicted by the Galaxy model at longitude $l=1^{\circ}$ for different latitudes $b$ and assuming different extinctions $A_{V}$

\begin{tabular}{lcc}
\hline$|b|\left(^{\circ}\right)$ & $A_{V}(\mathrm{mag})$ & Bulge $\left(\right.$ stars deg $\left.^{-2}\right)$ \\
\hline 0 & 4 & 40 \\
1 & 4 & 0 \\
& 3 & 140 \\
2 & 3 & 90 \\
& 2 & 3670 \\
3 & 3 & 80 \\
& 2 & 2880 \\
5 & 3 & 10 \\
& 2 & 1180 \\
\hline
\end{tabular}

Schultheis et al. (1999), using near-infrared star counts of the DENIS survey, computed the mean extinction at the distance of the bulge. The result is a high resolution 2D map of the interstellar extinction for $|b|<1.5^{\circ}$. According to this map, close to the Galactic plane $\left(b \leq 1^{\circ}\right)$ extinction is higher than four magnitudes in the $V$ band. This makes the bulge detection by the RAVE survey unlikely.

At higher latitudes, Schlegel et al. (1998) obtained a map of dust infrared emission from COBE and IRAS data, and derived the total extinction along the line of sight. Right in the plane the total extinction would be about twice the value undergone by bulge stars, the difference being due to extinction in the background. At latitudes $b \geq 2^{\circ}$, this value is a good estimate of the extinction at the distance of the bulge. Indeed, at this distance, the stars are above the Galactic plane and less affected by the interstellar matter which is confined to the plane. Using this map, we searched for regions in the Galaxy where extinction is low enough to allow RAVE to reach bulge stars, according to the stellar densities given in Table 1. These regions are plotted in Figure 3, where the different colours indicate different extinction values.

\section{The Bulge, as Seen by Gaia}

The Gaia satellite will be launched at the end of the decade (Perryman 2002). It will provide accurate positional, radial velocity, and photometric measurements for one billion stars, that is, about $1 \%$ of the Milky Way stellar content. It comprises two telescopes for astrometry and broad-band

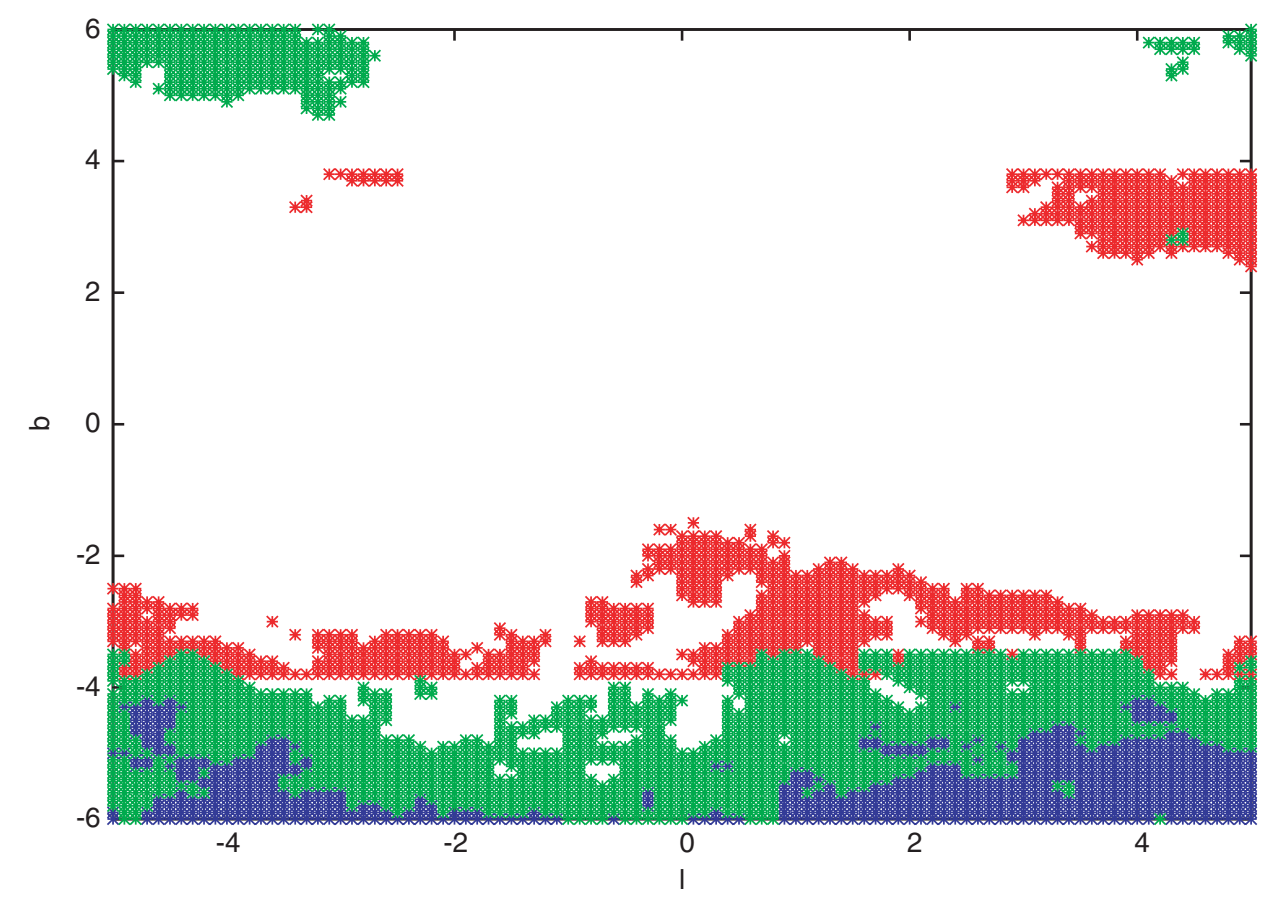

Figure 3 Coloured regions show where the predicted density of bulge stars observed by RAVE is not null. The values of the extinction considered to estimate the density are taken from Schlegel et al.'s (1998) map. Red: $A_{V}=3 \pm 0.5$. Green: $A_{V}=2 \pm 0.5$. Blue: $A_{V} \leq 1.5$. In higher extinction regions, the bulge is not reached (white regions). 
Table 2. Density of bulge stars and all stars as predicted by the Galaxy model at $l=1^{\circ}$ for different latitudes $b$ and assuming different extinctions $A_{V}$, up to $G=17$ (RVS) and $G=20$ (for MBP and BBP). Bold numbers indicate when crowding occurs

\begin{tabular}{|c|c|c|c|c|c|}
\hline \multirow{2}{*}{$\begin{array}{l}|b| \\
\left({ }^{\circ}\right) \\
0\end{array}$} & \multirow{2}{*}{$\begin{array}{c}A_{V} \\
(\mathrm{mag}) \\
6\end{array}$} & \multicolumn{2}{|c|}{$\begin{array}{l}\begin{array}{l}\text { Bulge } \\
\text { (stars deg } \\
G \leq 17 \\
G \leq 17\end{array} \\
\text { Total }\end{array}$} & \multicolumn{2}{|c|}{$\begin{array}{l}\text { Bulge } \\
\left.\text { (stars deg }{ }^{-2}\right) \\
\quad G \leq 20\end{array}$} \\
\hline & & 1154 & 14492 & 233454 & 513199 \\
\hline & 8 & 3 & 4908 & 49243 & 129879 \\
\hline & 15 & 0 & 2094 & 88 & 7876 \\
\hline \multirow[t]{4}{*}{1} & 5 & 7009 & 21826 & 358012 & 675607 \\
\hline & 6 & 1043 & 9978 & 220043 & 412072 \\
\hline & 8 & 2 & 3700 & 46523 & 101527 \\
\hline & 10 & 0 & 2421 & 14622 & 33637 \\
\hline \multirow[t]{4}{*}{2} & 4 & 21018 & 41479 & 406818 & 689042 \\
\hline & 6 & 876 & 7254 & 185476 & 292397 \\
\hline & 8 & 2 & 3040 & 39437 & 74032 \\
\hline & 10 & 0 & 2212 & 12239 & 25587 \\
\hline \multirow[t]{3}{*}{3} & 3 & 36002 & 62451 & 414002 & 695685 \\
\hline & 5 & 4430 & 11028 & 227732 & 310952 \\
\hline & 8 & 2 & 2616 & 29586 & 53130 \\
\hline \multirow[t]{3}{*}{5} & 1 & 81468 & 126263 & 1107570 & 1599950 \\
\hline & 3 & 14138 & 27207 & 156438 & 285878 \\
\hline & 5 & 1910 & 5812 & 85872 & 118868 \\
\hline
\end{tabular}

photometry (BBP) up to $G=20,{ }^{2}$ and one telescope for medium-band photometry (MBP) up to $G=20$ and radial velocities (RVS instrument) up to $G=17$. Each star will be observed one hundred times during the time of the mission, allowing the determination of proper motions.

On the sky mappers, detection problems may occur if the observed field is crowded. The estimated crowding limit is $10^{6}$ stars $\mathrm{deg}^{-2}$ for the BBP (Jordi et al. 2003) and $10^{5}$ stars deg $^{-2}$ (Høg 2002) for the MBP with $G \leq 20$. Radial velocities could be obtained with a good precision up to densities of about $20000 \mathrm{stars} \mathrm{deg}^{-2}$ with $G \leq 17$ (T. Zwitter \& D. Katz 2003, private communication). However, these estimates are still being investigated. Crowding may occur in regions with low extinction in the bulge direction.

Table 2 gives the stellar density predicted by the Besançon model for longitude $l=1^{\circ}$, at different latitudes $b$, and assuming different interstellar absorptions $A_{V}$. The columns with $G \leq 17$ are the bulge and total stellar densities as seen by the RVS, while the columns with $G \leq 20$ are those for the MBP and BBP instruments.

The total stellar density allows us to check whether the field is crowded or not. Numbers in bold correspond to fields and extinctions where crowding will be an issue. In the bulge direction, crowding will be a major obstacle to the observation with MBP (most of the values of total stellar density are greater than $10^{5} \mathrm{stars} \mathrm{deg}^{-2}$ for $G \leq 20$ ). However, proper motions still will be measured with BBP.

\footnotetext{
${ }^{2} G$ is the photometric band used by the Gaia sky mappers. It is close to the $V$ magnitude for stars with $V-I=0$. Note that it is now changed in the new design of the payload. The difference can be as large as one magnitude, depending on $V-I$.
}

Figures 4 and 5 show the regions in the Galaxy where Gaia should be able to measure radial velocities with the RVS, and proper motions with the BBP. These plots were obtained using the values of the extinction from Schultheis et al. (1999) (black) and from Schlegel et al. (1998) (grey). The non-continuity between these two maps is due to the fact that the methods used to obtain them are different. At low latitudes $\left(|b|<2^{\circ}\right)$, the total extinction given by Schlegel et al. (1998) overestimates the extinction at the distance of the bulge. Therefore, stars are fainter and less accessible to observation compared to what is expected considering Schultheis et al.'s (1999) extinction map.

\section{Discussion}

Using the stellar population synthesis model of Besançon, and based on available extinction maps, we predicted that ongoing or future large-scale surveys RAVE and Gaia will be able to bring a large amount of data for bulge stars. RAVE should be able to measure radial velocities in the bulge for $|b| \geq 2^{\circ}$, particularly in the southern hemisphere where the extinction is lower. Gaia will survey the Galaxy at a deeper magnitude, but the detection of objects can then become impossible in crowded fields. In that case, regions with high extinction can help in bypassing this problem. Radial velocities should be measured with the spectrograph RVS onboard Gaia at Galactic latitudes as low as $0.5^{\circ}$ in some parts of the bulge. Moreover, the outer part of the bulge will be easily reached in the northern hemisphere, while in the southern hemisphere, crowding will be problematic due to lower extinction. Gaia should also obtain proper motions with the BBP in most of the bulge regions, except in the plane and close to the Galactic centre where extinction is the highest. 


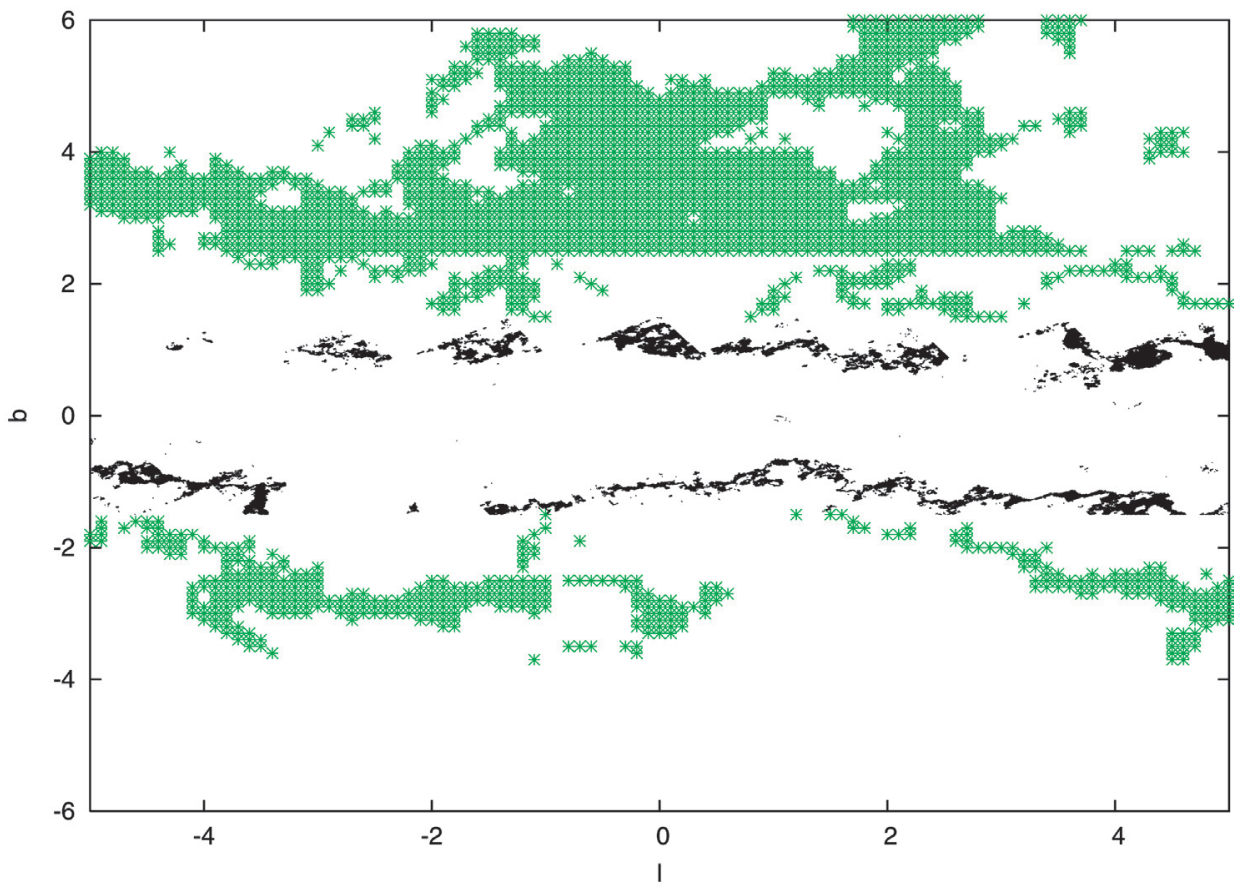

Figure 4 Regions in the Galaxy where bulge stars are expected to be observed with RVS. The grey asterisks indicate when the values of the extinction we used to compute the stellar density are from Schlegel et al. (1998), and the black dots are based on extinction data from Schultheis et al. (1999). In white regions, the bulge cannot be observed due to high extinction close to the Galactic plane $\left(|b|<1^{\circ}\right.$ ) or due to crowding at higher latitudes (around $b \simeq 5^{\circ}$ and $b \simeq-4^{\circ}$ ), in particular in the southern hemisphere.

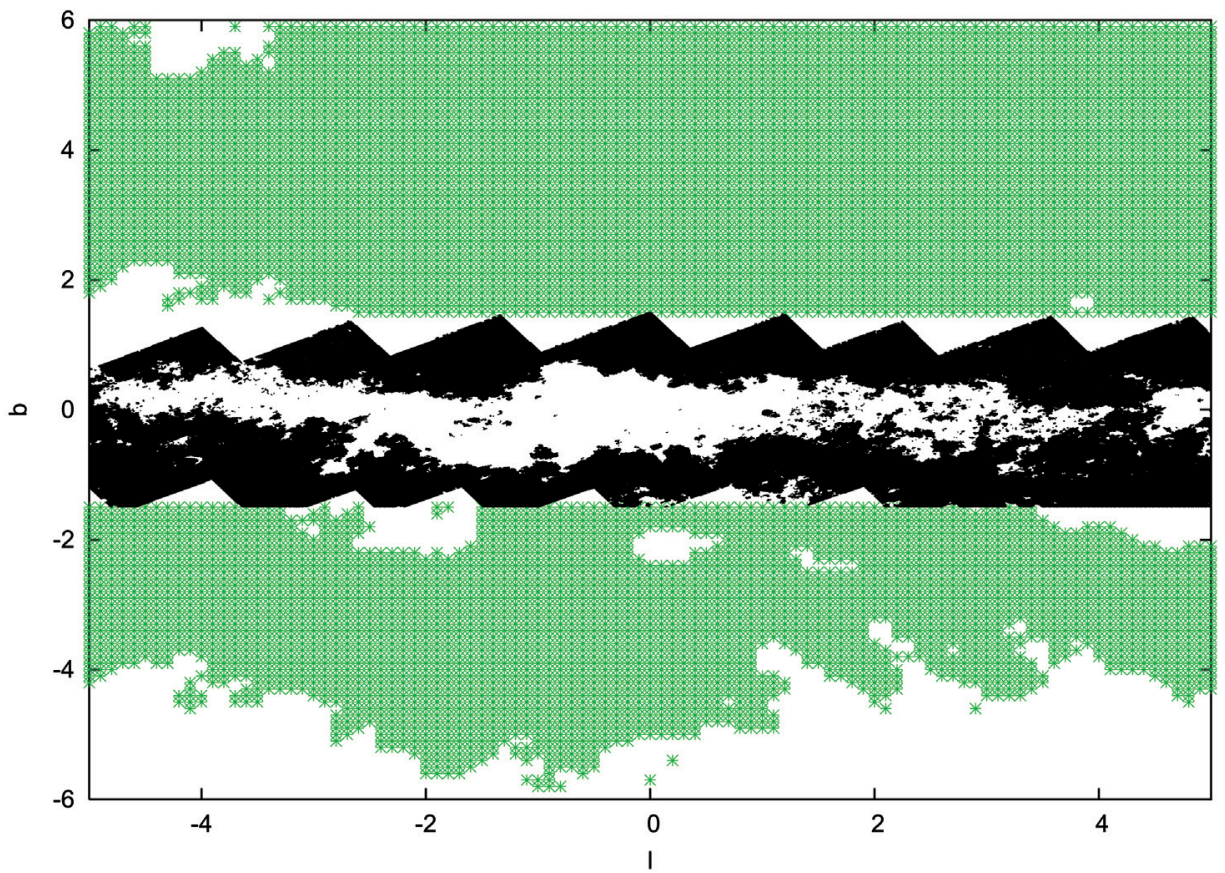

Figure 5 Same as Figure 4 for the BBP. In white regions, the bulge cannot be observed due to high extinction close to the Galactic plane $\left(|b|<0.5^{\circ}\right)$ or due to crowding at higher latitudes, in particular in the southern hemisphere (around $b \simeq-5^{\circ}$ ).

The result depends on the galaxy model used. This model has been obtained by adjusting density parameters of the bulge and inner disk with a set of near-infrared star counts in almost a hundred windows. The quality of fit is assessed by a $\chi^{2}$ score, which is less than $3 \sigma$ in the majority of the windows. Hence we estimate the model to be accurate to about $15 \%$ in counts, except in particular regions (star forming regions, clusters, etc.). 
The separation of bulge stars from other populations is not, however, an easy task, particularly with the disk giants. If proper motions are measured (in the case of Gaia observations), the reduced proper motions can be used to isolate the bulge stars. However, the Galaxy model shows that only about half of the bulge stars can be well separated up to $G=20$. When only radial velocities are available, it seems impossible to distinguish bulge stars from other populations, except for a few stars in the tails of the radial velocity distribution. Nevertheless, different populations are expected to show different gradients from field to field. Therefore, detailed modelling is required if one wants to extract the information.

Furthermore, radial velocities data are available in the bulge directions (Ibata \& Gilmore 1995; Minniti 1996; Minniti et al. 1996; Tiede \& Terndrup 1999). It is necessary to compare these data with the Besançon model of the Galaxy in order to refine our kinematic parameters. Finally, the works cited above also give metallicity measurements and could be used in adjusting the bulge metallicity parameter with the Galaxy model. This parameter will be available from both Gaia and RAVE surveys.

\section{References}

Alard, C. 2003, A\&A, preprint astro-ph/0306624

Høg, E. 2002, Gaia report GAIA-CUO-115

Ibata, R. A., \& Gilmore, G. F. 1995, MNRAS, 275, 605
Jordi, C., Figueras, F., Carrasco, J. M., \& Knude, J. 2003, Gaia report UB-PWG-009

Minniti, D. 1996, ApJ, 459, 579

Minniti, D., Liebert, J., Olszewski, E. W., \& White, S. D. M. 1996, AJ, 112, 590

Parker, Q. A., \& Phillipps, S. 2001, in The New Era of Wide Field Astronomy, ASP Conf. Series 232, eds. R. Clowes, A. Adamson, \& G. Bromage (San Francisco: ASP), 38

Perryman, M. A. C. 2002, Ap\&SS, 280, 1

Peyaud, A. E., Parker, Q. A., Acker, A. A., \& Bond, I. A. 2003, in White Dwarfs: Galactic and Cosmologic Probes, 25th meeting of the IAU, Joint Discussion 5, 16-17 July 2003, Sydney

Picaud, S., Chereul, E., Robin, A. C., \& Garzón, F. 2003, Ap\&SS, 284,523

Robin, A. C., Reylé, C., Derrière, S., \& Picaud, S. 2003, A\&A, 409, 523

Schlegel, D. J., Finkbeiner, D. P., \& Davis, M. 1998, ApJ, 500, 525

Schultheis, M., et al. 1999, A\&A, 349, L69

Steinmetz, M. 2003, in Gaia Spectroscopy, Science, and Technology, ASP Conf. Series 298, ed. U. Munari (San Francisco: ASP), 381 Sumi, T., et al. 2004, MNRAS, 348, 1439

Tiede, G. P., \& Terndrup, D. M. 1999, AJ, 118, 895 